Mathematical Research Letters 6, 429-437 (1999)

\title{
FOUR-MANIFOLD GEOGRAPHY AND SUPERCONFORMAL SYMMETRY
}

\author{
Marcos Mariño, Gregory Moore, and Grigor Peradze
}

\begin{abstract}
A compact oriented 4-manifold is defined to be of "superconformal simple type" if certain polynomials in the basic classes (constructed using the Seiberg-Witten invariants) vanish identically. We show that all known 4-manifolds of $b_{2}^{+}>1$ are of superconformal simple type, and that the numerical invariants of 4-manifolds of superconformal simple type satisfy a generalization of the Noether inequality. We sketch how these phenomena are predicted by the existence of certain four-dimensional superconformal quantum field theories.
\end{abstract}

\section{Introduction}

On several occasions insights into the physics of quantum field theory and string theory have suggested new results and techniques in mathematics, particularly in geometry and topology. One of the main focal points of activity in physics in the past few years has been the study of superconformal field theories in four dimensions. In this letter we show that some of the recent progress in physics leads to new results on the geography of four-manifolds.

This letter is primarily intended for a mathematical audience. The physical motivation for our results is summarized briefly in section five. A more extensive account of this work can be found in [13].

\section{SST Manifolds}

Let $X$ be a compact, oriented four-manifold with $b_{2}^{+}>1$. In this letter we will address the relation between the classical numerical invariants of $X$ :

$$
\begin{aligned}
\chi_{h} & :=\frac{\chi+\sigma}{4}=\frac{1-b_{1}+b_{2}^{+}}{2}, \\
c_{1}^{2} & :=2 \chi+3 \sigma=4-4 b_{1}+5 b_{2}^{+}-b_{2}^{-},
\end{aligned}
$$

and its Seiberg-Witten invariant, which is a map SW $: \operatorname{Spin}^{c}(X) \rightarrow \mathbb{Z}$ from the $\operatorname{Spin}^{c}$ structures of $X$ to the integers. SW is a topological invariant of $X$ defined using a signed sum over solutions to the monopole equations [19]. We will

Received March 1, 1999. 
identify $\operatorname{Spin}^{c}(X)$ with elements of $H^{2}(X, \mathbb{Z})$ congruent to $w_{2}(X) \bmod 2^{1}$, and say that $x \in \operatorname{Spin}^{c}(X)$ is a basic class if $\operatorname{SW}(x) \neq 0$. In this letter we will assume that $X$ is of (Seiberg-Witten) simple type, i.e., if $x^{2} \neq c_{1}^{2}$ then $\mathrm{SW}(x)=0$. If $X$ is of simple type and the SW invariant is not trivial, then $X$ must obey the Noether condition, i.e., $\chi_{h} \in \mathbb{Z}$. Notice that, if $X$ is a complex surface, then $\chi_{h}$ is in fact the holomorphic Euler characteristic, and $c_{1}^{2}$ is the square of the first Chern class of the holomorphic tangent bundle.

Choose an integral lifting $v$ of $w_{2}(X)$ and consider the twisted SW series:

$$
\mathrm{SW}_{X}^{w_{2}(X)}(z):=\sum_{x}(-1)^{\frac{v^{2}+v \cdot x}{2}} \mathrm{SW}(x) \mathrm{e}^{z x} .
$$

This is a finite sum [19]. A change of lifting $v \rightarrow \tilde{v}$ alters $\mathrm{SW}_{X}^{w_{2}(X)}$ by a sign $(-1)^{\left(\frac{v-\tilde{v}}{2}\right)^{2}}$.

Definition 2.1. Let $X$ be a compact, oriented 4-manifold of simple type with $b_{2}^{+}>1$. We say that " $X$ is $\operatorname{SST}^{\prime}$ if $\operatorname{SW}_{X}^{w_{2}(X)}(z)$ has a zero at $z=0$ of order $\geq \chi_{h}-c_{1}^{2}-3$.

The phrase " $X$ is SST" is short for " $X$ is of superconformal simple type." The terminology comes from the physical motivation discussed at the end of this letter. The SST condition is equivalent to the condition that either $c_{1}^{2}-\chi_{h}+3 \geq 0$ or

$$
\sum_{x}(-1)^{\frac{v^{2}+v \cdot x}{2}} \mathrm{SW}(x) x^{k}=0, \quad k=0, \ldots, \chi_{h}-c_{1}^{2}-4,
$$

where $x^{k}$ is naturally understood as an element in $\left(\operatorname{Sym}^{k}\left(H^{2}(X, \mathbb{Z})\right)^{*}\right.$ acting through the intersection form. Notice that, if $\chi_{h}+\sigma$ is even (odd), the expressions of the form (3) with $k$ odd (even) are automatically zero. This is easily proved using the fact that, if $x$ is a basic class, then so is $-x$, and

$$
(-1)^{\frac{v^{2}-v \cdot x}{2}} \mathrm{SW}(-x)=(-1)^{\chi_{h}+\sigma}(-1)^{\frac{v^{2}+v \cdot x}{2}} \mathrm{SW}(x),
$$

as one easily checks using the $\mathrm{Wu}$ formula and the behavior of the $\mathrm{SW}$ invariants under the involution $x \rightarrow-x[19]$. Therefore, there are only $\left[\frac{1}{2}\left(\chi_{h}-c_{1}^{2}\right)\right]-1$ nontrivial equations in (3), where [.] is the greatest integer function.

\section{Two theorems about SST manifolds}

In this section, we will use the known behavior of the Seiberg-Witten invariants to state some properties of SST manifolds. The detailed proofs of Theorems 3.1 and 3.2 can be found in [13]. We only make some brief remarks on the proofs here.

\footnotetext{
${ }^{1}$ If the second cohomology has 2 -torsion, then there is not a one-to-one correspondence between $\operatorname{Spin}^{c}$ structures and integer liftings of $w_{2}(X)$. We will assume in what follows that there is no 2-torsion in cohomology. This happens, in particular, when $X$ is simply-connected.
} 
Theorem 3.1. The property " $X$ is $S S T$ " is preserved by blowup, fiber sum along c-embedded tori, knot surgery, and generalized log transforms.

The proof of this theorem is a simple consequence of the known behavior of the SW invariants under these operations [4][5][6][15]. The blowup is particularly instructive. If $\widehat{X}=X \sharp \overline{\mathbb{C} P^{2}}$ denotes the blownup manifold, with exceptional divisor $E$, one has

$$
\mathrm{SW}_{\widehat{X}}^{w_{2}(\widehat{X})}(z)=-2 \sinh (z E) \mathrm{SW}_{X}^{w_{2}(X)}(z),
$$

where we choose the lift of $w_{2}(\widehat{X})$ to be $\hat{v}=v+E$. Using $c_{1}^{2}(\widehat{X})=c_{1}^{2}(X)-1$, it immediately follows that if $X$ is SST then $\widehat{X}$ is SST. This computation shows that the sign factor in (2) is crucial.

The proof for the fiber sum along c-embedded tori is based on the gluing formulae of [4][5][6][15]. Using these formulae, one shows that, if $X=X_{1} \sharp T_{1}=T_{2} X_{2}$ is the fiber sum of two SST manifolds, then

$$
\mathrm{SW}_{X}^{w_{2}(X)}(z)= \pm 4(\sinh z T)^{2} \mathrm{SW}_{X_{1}}^{w_{2}\left(X_{1}\right)}(z) \cdot \mathrm{SW}_{X_{2}}^{w_{2}\left(X_{2}\right)}(z)
$$

where the \pm sign depends on the choice of the liftings and $T$ is the common torus. The proof that $X$ is SST is easy arithmetic based on (6).

The case of knot surgery also follows from [5]. Finally, we consider the generalized $\log$ transform for manifolds with a cusp neighborhood. This is done by doing $p-1$ blowups, $X \rightarrow X \sharp(p-1) \overline{\mathbb{C} P^{2}}$, and then performing a rational blowdown along a $C_{p}$ configuration. See [6][16][9] for details. Again, the proof is simple given the known behavior of the SW invariants under these operations.

Theorem 3.2. If $X$ is a compact complex surface of $b_{2}^{+}>1$ then $X$ is SST.

Proof. Since property SST is preserved by blowup, we can restrict to minimal complex surfaces. Using the Kodaira-Enriques classification we need only consider elliptic surfaces and surfaces of general type. For minimal surfaces of general type, the property SST is a consequence of the Noether inequality. For minimal elliptic fibrations with no multiple fibers the computation of the twisted series 0 follows from [7][8][2] and is:

$$
\mathrm{SW}_{X}^{w_{2}(X)}(z)=(2 \sinh (z f))^{\chi_{h}+2 g-2},
$$

Here $g \geq 0$ is the genus of the base of the fibration and $f$ is the class of the fiber. Taking into account that $c_{1}^{2}=0$ the SST property follows immediately. In this computation we used the lift $v=c_{1}(K)$, with $K$ the canonical bundle of the elliptic surface. Since the SST property is preserved by generalized log transforms it follows that any minimal elliptic surface is SST.

Most of the recent constructions of "exotic" manifolds (symplectic but noncomplex manifolds or irreducible nonsymplectic manifolds) are based on the 
constructions we have considered, and use complex surfaces as their building blocks. Therefore, they are all SST. For example, the SST property is also preserved by rational blowdowns of tautly embedded $C_{n-2}$ configurations in the simply connected elliptic fibrations $E(n)$ (for $n \geq 4$ ). In particular, after a rational blowdown along one $C_{n-2}$ in $E(n)$, one obtains an interesting exotic manifold $Y(n)$. Using [4][6] one finds that $c_{1}^{2}=\chi_{h}-3$ for $Y(n)$, so it is SST. Based on the above results and the examination of many other examples, we accordingly formulate the following:

Conjecture 3.3. Every compact, oriented 4-manifold of $b_{2}^{+}(X)>1$ is SST.

We will briefly explain the physical reasons for this conjecture in section 5 . It would be desirable to have more results along the lines of this section for other constructions of four-manifolds, such as arbitrary rational blowdowns or fiber sums along surfaces of genus $g>1$. This may help to provide a mathematical proof of the above conjecture.

\section{Bounds on the number of basic classes for SST manifolds}

One of the most important properties of SST manifolds is that, if they support any basic classes at all, then there is a lower bound on the number of such classes in terms of the classical numerical invariants of $X$. A corollary of this lower bound is a generalized Noether inequality that relates the values of the numerical invariants $c_{1}^{2}, \chi_{h}$, to the number of basic classes. ${ }^{2}$ This gives a deep relation between the Seiberg-Witten invariants and the geography of four-manifolds.

Let $\mathcal{B}_{X}$ denote the set of basic classes of $X$. If $x$ is a basic class, then so is $-x$, and it is convenient to mod out by this involution when counting the number of basic classes. We will then say that $X$ has $B$ basic classes if the set $\mathcal{B}_{X} /\{ \pm 1\}$ consists of $B$ elements.

Theorem 4.1 [Generalized Noether inequality]. Let $X$ be SST. If $X$ has $B$ distinct basic classes and $B>0$, then

$$
B \geq\left[\frac{\chi_{h}-c_{1}^{2}}{2}\right]
$$

In particular, $c_{1}^{2} \geq \chi_{h}-2 B-1$.

The theorem is a direct consequence of the conditions (3) for SST manifolds. The detailed proof can be found in [13]. The bound (8) is in fact sharp since it is saturated by the simply-connected elliptic fibrations $E(n)$. It follows from Theorem 4.1 that manifolds with only one basic class must satisfy $c_{1}^{2} \geq \chi_{h}-3$. This inequality is saturated, as we have noted, by the exotic manifolds $Y(n)$

\footnotetext{
${ }^{2}$ We call the inequality above a generalized Noether inequality because of its similarity to the Noether inequality for minimal surfaces of general type, which asserts that $c_{1}^{2} \geq 2 \chi_{h}-6$. Notice, however, that our inequality has half the slope of the usual Noether inequality.
} 
obtained by rational blowdown of $E(n)$ [6]. All known constructions, e.g., those found in [6], of manifolds with one basic class satisfy our inequality.

Given a metric on $X$ one can also give an upper bound on $B$ in terms of certain Riemannian functionals, using the ideas in [19]. Thus, there exist topological lower bounds on these Riemannian functionals.

\section{Sketch of the relation to superconformal field theory}

The above results and definitions are motivated by the existence of superconformal fixed points in certain physical theories. In this section, we summarize the main physical ideas behind the above results. Further discussion can be found in [13].

Donaldson theory can be interpreted as a "topologically twisted" version of an $\mathcal{N}=2$ supersymmetric Yang-Mills theory [20]. The twisting procedure, which can be understood as a redefinition of the coupling to gravity, produces quantum field theories which are formally "topological," in the sense that the correlation functions do not depend on the metric of the four-manifolds. In this way, certain correlation functions of the twisted $\mathcal{N}=2$ theory are identified with Donaldson invariants. These correlation functions can be assembled into a generating function known as the Donaldson-Witten partition function and denoted by $Z_{D W}$.

The data specifying the general $d=4, \mathcal{N}=2$ supersymmetric gauge theory is a choice of a compact Lie group $G$ (the gauge group), a finite dimensional representation $R$ (the matter hypermultiplet representation) and a $G$-invariant tensor in $R \otimes R^{*}$ (the masses of the matter hypermultiplets). The procedure of "topological twisting" only depends on the structure of the $\mathcal{N}=2$ supersymmetry algebra, so any of these models can be twisted to obtain a topological field theory. A particularly interesting case is the topological theory defined by the gauge group $S U(2)$, with one matter multiplet in the fundamental representation of $S U(2)$, denoted $\mathbf{2}$. This theory defines the moduli space associated to the nonabelian monopole equations, and it can be analyzed following the lines of Donaldson theory. (See [11] for a review from the point of view of topological field theory, and [3] for a recent review of rigorous results.) In particular, one can formally define topological invariants using intersection theory in the moduli space of solutions. An interesting aspect of this theory is that the mass term can be interpreted as the formal parameter of the equivariant cohomology of the moduli space with respect to a $U(1)$ group action [10]. Therefore, the correlation functions of this topological theory, considered as functions of the mass, formally compute the equivariant intersection theory on the moduli space of nonabelian monopoles. More generally, for the theory specified by $G, R$ the masses $m$ are the parameters for the equivariant cohomology of a group action on the moduli space of the corresponding (nonabelian) monopole equations.

The low-energy dynamics of the $S U(2) \mathcal{N}=2$ supersymmetric gauge theories was solved by Seiberg and Witten in [18] in terms of certain families of elliptic curves. In the case when there are no matter multiplets Witten used this to 
obtain the topological correlation functions, i.e., the Donaldson invariants, in terms of the SW invariants [19]. The result of [19] can be generalized to the theories with matter, as explained in [14], and one obtains a generalization of Witten's formula, i.e., an exact expression for the generating function of equivariant intersection numbers, $Z_{D W}(p, S ; m)$. In this function, $p, S$ correspond to (equivariant) differential forms of degree four and two, respectively, on the corresponding moduli space, and generalize the usual observables of DonaldsonWitten theory.

We now describe the function $Z_{D W}(p, S ; m)$ for the case $G=S U(2), R=\mathbf{2}$ in more detail. The Seiberg-Witten family of elliptic curves is parametrized by $(u, m) \in \mathbb{C}^{2}$ and given by:

$$
y^{2}=x^{2}(x-u)+2 m x-1 .
$$

The curve is easily put into standard form $y^{2}=4 x^{3}-g_{2} x-g_{3}$, with $g_{2}(u ; m)=$ $\frac{4}{3}\left(u^{2}-6 m\right), g_{3}(u ; m)=\frac{1}{27}\left(8 u^{3}-72 m u+108\right)$, and discriminant $\Delta(u ; m)=$ $g_{2}^{3}-27 g_{3}^{2}$. This discriminant is a cubic in $u$ and has three roots $u_{j}(m), j=1,2,3$. For generic, but fixed, values of $m$ one of the periods of (9) goes to infinity as $u \rightarrow u_{j}$ while the other period, $\varpi_{j} \equiv \varpi\left(u_{j}(m) ; m\right)$ remains finite, and in fact is given by $\left(\varpi_{j}\right)^{2}=g_{2} /\left(36 g_{3}\right)$. The Donaldson- Witten partition function on a manifold $X$ of simple type and $b_{2}^{+}(X)>1$ is given by a sum over the singular fibers of the Weierstrass family (9) and over the basic classes of $X$ :

$$
\begin{aligned}
Z_{D W}(p, S ; m)= & k \sum_{j=1}^{3}\left(\frac{g_{2}^{3}\left(u_{j}(m) ; m\right)}{\Delta^{\prime}\left(u_{j}(m) ; m\right)}\right)^{\chi_{h}}\left(\varpi_{j}(m)\right)^{7 \chi_{h}-c_{1}^{2}} \\
& \sum_{x} \mathrm{SW}(x)(-1)^{\left(v^{2}+v \cdot x\right) / 2} \exp \left[2 p u_{j}+S^{2} T_{j}-i \frac{(S, x)}{2 \varpi_{j}}\right]
\end{aligned}
$$

Here $\Delta^{\prime}=\frac{\partial}{\partial u} \Delta, T_{j}=-\frac{1}{24}\left(\left(\varpi_{j}\right)^{-2}-8 u_{j}\right)$, and $k$ is a nonvanishing constant, independent of $p, S, m$. Similar expressions hold for gauge groups $G$ of rank bigger than one [12].

At first sight, (10) and its generalizations are disappointing, since they imply that the more general topological theories and moduli equations obtained from general supersymmetric gauge theories do not contain new topological information. While (10) and its generalizations seem disappointing, defeat can be turned into victory by using known properties of the physical theories to learn about the SW invariants. From the mathematical viewpoint, the parameters $m$ of equivariant cohomology are formal expansion parameters, but from the physical viewpoint it is clear that we should regard $Z_{D W}(p, S ; m)$ as nontrivial locally analytic functions of $m$. At certain points of the moduli space, i.e., at special values of $m$, the low-energy SW theory is in fact a nontrivial superconformal field theory (i.e., a conformal field theory with $\mathcal{N}=2$ supersymmetry) [1]. We now describe how the analytic structure of $Z_{D W}(p, S ; m)$ at such points can lead to the nontrivial predictions for topology described above. 
Let us summarize some facts about the SW curve for $G=S U(2), R=\mathbf{2}$. Substituting $m=\frac{3}{2}+z, u=3+2 z+\delta u$ into (9) and taking $z, \delta u$ small leads to the curve

$$
y^{2}=x^{3}-2 z x-\delta u
$$

The family (11) develops a cusp singularity as $z, \delta u \rightarrow 0$. Indeed, when $z \rightarrow 0$, two of the roots of $\Delta(u ; m)=0$, call them $u_{ \pm}(m)$, coincide. By scaling one finds $\delta u_{ \pm} \sim z^{3 / 2}$. In the limit $z \rightarrow 0$ the period $\varpi_{ \pm}$diverges as $z^{-1 / 4}$, while $g_{2}\left(u_{ \pm}(m) ; m\right) \sim z$ and $\Delta^{\prime}\left(u_{ \pm}(m) ; m\right) \sim \delta u_{ \pm} \sim z^{3 / 2}$. At the third singularity all the various factors in (10) are given by nonvanishing analytic series in $z$, but, evidentally, the contributions from $u_{ \pm}(m)$ contain factors which are diverging or vanishing as $z \rightarrow 0$. What can we say about the behavior of the complete function $Z_{D W}$ as $z \rightarrow 0$ ?

The detailed expansion of $Z_{D W}$ as a power series in $z$ is discussed at length in [13]. An easy consequence of $(4)$ is that $Z_{D W}(z)$ is a Laurent series in integral powers of $z$. Using the facts from the previous paragraph one checks that the Laurent series (10) has at most a finite order pole of order $z^{\left(c_{1}^{2}-\chi_{h}\right) / 4}$. Thus, if $c_{1}^{2}-\chi_{h}<0$ there could be a pole in $z$. However, the physical origin of $Z_{D W}$ leads to a powerful and well-founded principle:

If $X$ is compact, and the moduli space of solutions to the relevant abelian monopole equations is compact, then $Z_{D W}$ must be a regular analytic function of $z$ near $z=0$.

The reason for this principle is that divergences in topological field theory, even at superconformal points, can only arise from infrared divergences in spacetime or in moduli space. Since $X$ is compact there are no divergences in spacetime. If the moduli space is compact then $Z_{D W}$ cannot diverge.

If $c_{1}^{2}-\chi_{h}<-3$ then the coefficients of the would-be poles in the $z$-expansion are polynomials in $p, S^{2}$, and $(S, x)$. The regularity of $Z_{D W}$ at $z=0$ thus implies nontrivial constraints on these quantities. Unfortunately, the detailed expansion described in [13] is somewhat complicated. Nevertheless, since $\varpi_{ \pm} \sim z^{-1 / 4}$ it follows from expanding the exponential in (10) that if the sum rules (3) are satisfied then $Z_{D W}$ is indeed analytic at $z=0$. This sufficient condition is the SST condition. As we have checked above, all known 4-manifolds of $b_{2}^{+}>1$ satisfy the condition.

Close examination of the Laurent expansion in $z$ of (10) reveals that the SST property is sufficient, but not necessary, for regularity [13]. It is thus logically possible that there exist 4-manifolds of simple type which are not SST, and yet do not violate our physical principle of regularity. However, the full statement of conditions for regularity of $Z_{D W}$ is rather involved, and we find this possibility extremely unlikely, both from the point of view of quantum field theory and from the point of view of four-manifold topology. It is worth noting that, even if we do drop the assumption that $X$ is SST, regularity of $Z_{D W}$ implies that if $X$ has 
one basic class then it satisfies the generalized Noether inequality $c_{1}^{2} \geq \chi_{h}-3$ [13].

One can probably even relax the condition that $X$ is of simple type. Every 4-manifold of $b_{2}^{+}>1$ is of generalized simple type [14]. Using equations (7.12) and (11.27) of [14] it should be possible to extend the above results to those (hypothetical) manifolds of $b_{2}^{+}>1$ which are not of simple type.

It would be very interesting to see whether the reasoning we have described here leads to further results in topology using other $\mathcal{N}=2$ superconformal fixed points.

\section{Acknowledgements}

We would like to thank E. Calabi, D. Freed, R. E. Gompf, T.J. Li and E. Witten for very helpful discussions and correspondence. This work is supported by DOE grant DE-FG02-92ER40704.

\section{References}

[1] P.C. Argyres, M.R. Plesser, N. Seiberg, and E. Witten, New $\mathcal{N}=2$ superconformal field theories in four dimensions, Nucl. Phys. B 461 (1996), 71-84, hep-th/9511154.

[2] R. Brussee, The canonical class and the $C^{\infty}$ properties of Kähler surfaces, New York J. Math. 2 (1996), 103-146.

[3] P.M.N. Feehan and T.G. Leness, PU(2) monopoles and relations between four-manifold invariants, Topology Appl. 88 (1998), 111-145, dg-ga/9709022.

[4] R. Fintushel and R.J. Stern, Constructions of smooth four-manifolds, Proceedings of the International Congress of Mathematicians, Vol. II (Berlin, 1998), Doc. Math. Extra Vol. II, $443-452$.

[5] Knots, links, and four-manifolds, Inv. Math. 134 (1998), 363-400, dg-ga/9612004.

[6] , Rational blowdowns of smooth four-manifolds, J. Differential Geom. 46 (1997), 181-235, alg-geom/9505018.

[7] R. Friedman and J.W. Morgan, Algebraic surfaces and Seiberg-Witten invariants, J. Alg. Geom. 6 (1997), 445-479, alg-geom/9502026.

[8] —, Obstruction bundles, semiregularity, and Seiberg-Witten invariants, alg-geom/9509007.

[9] R.E. Gompf and A.I. Stipsicz, 4-Manifolds and Kirby calculus, Amer. Math. Soc. (1999).

[10] J.M.F. Labastida and M. Mariño, Twisted $\mathcal{N}=2$ supersymmetry with central charge and equivariant cohomology, Comm. Math. Phys. 185 (1997), 37-71, hep-th/9603169.

[11] M. Mariño, The geometry of supersymmetric gauge theories in four dimensions, hepth/9701128.

[12] M. Mariño and G. Moore, The Donaldson-Witten function for gauge groups of rank larger than one, Commun. Math. Phys. 199 (1998), 25-69, hep-th/9802185.

[13] M. Mariño, G. Moore and G. Peradze, Superconformal invariance and the geography of four-manifolds, hep-th/9812055.

[14] G. Moore and E. Witten, Integration over the u-plane in Donaldson theory, Adv. Theor. Math. Phys. 1 (1997), 298-387.

[15] J.W. Morgan, T.R. Mrowka, and Z. Szabó, Product formulas along $T^{3}$ for Seiberg-Witten invariants, Math. Res. Lett. 4 (1997), 915-929.

[16] J. Park, The geography of irreducible four-manifolds, Proc. Amer. Math. Soc. 126 (1998), 2493-2503. 
[17] N. Seiberg and E. Witten, Electric-magnetic duality, monopole condensation, and confinement in $\mathcal{N}=2$ supersymmetric Yang-Mills theory, Nucl. Phys. B 426, 19-52, hepth/9407087.

[18] Monopoles, duality and chiral symmetry breaking in $\mathcal{N}=2$ supersymmetric QCD, Nucl. Phys. B 431 (1994), 485-486, hep-th/9408099.

[19] E. Witten, Monopoles and four-manifolds, Math. Res. Lett. 1 (1994), 769-796, hepth/9411102.

[20]

Department of Physics, Yale University, New Haven, CT 06520, USA

E-mail address: marcos.marino@yale.edu, moore@castalia.physics.yale.edu, grigor.peradze@yale.edu 